\title{
Prevalence and Symptomatic Correlation of Vitamin D Deficiency in Individuals Visiting for an Annual Health Check-up - A Cross-sectional Study
}

\author{
Ashish Kumar Maurya ${ }^{1 *}$, Rakhi $^{2}$ \\ ${ }^{1}$ Department of Preventive Health Apollo medics hospital, Lucknow, UP, India \\ ${ }^{2}$ Department of Radiology, Sahara Hospital, Lucknow, UP India
}

\begin{abstract}
Vitamin D plays a crucial role in electrolytic reabsorption and calcium homeostasis, thereby maintaining skeletal and extra-skeletal integrity. In addition, the literature establishes its potency to prevent insulin-dependent diabetes, cardiovascular diseases, malignancies, and various autoimmune disorders. Therefore, determining vitamin $D$ deficient states and its correlation with systemic factors plays a notable role in clinical medicine. The present cross-sectional study was conducted to determine the Prevalence and symptomatic correlation of vitamin D deficiency in individuals visiting an annual health check-up at a quaternary care hospital, Lucknow, Uttar Pradesh, India. The study stipulated the prevalence rate of serum vitamin D deficiency levels was $91 \%$ among healthy individuals. The overall mean value of serum vitamin D was $17.54 \mathrm{ng} / \mathrm{mL}$. With $91.2 \%$ symptomatic individuals, the mean age of patients was $48.08 \pm 10.33$ years, with the majority of males $(61.53 \%)$. The mild, moderate, and severe grades of vitamin D deficiency were seen in $32.53 \%, 53.01 \%$, and $14.45 \%$ of study participants, respectively. On applying Pearson correlation, there is a strong negative correlation between the total symptom score (12.98) and serum vitamin D levels with an $R$ value of -0.788. The study reveals the higher prevalence rate of vitamin $D$ deficiency among apparently healthy participants. The associated symptoms among moderate and severe vitamin D deficient study participants indicate the alarming stage in the country. The study concluded the need for comprehensive public health programs concerning the advantages of sun exposure and sufficient intake of fortified resources in the 21st-century lifestyle.
\end{abstract}

Keywords: Bone pain, Cholecalciferol, Depression, Muscle pain, Prevalence, Symptoms, Vitamin D deficiency.

\section{Introduction}

Vitamin D, as a micronutrient, plays a variety of major and minor roles in maintaining the overall systemic health of individuals, including electrolytic reabsorption, muscle contraction, calcium, and phosphorous homeostasis, and even affects the immune system, especially $\mathrm{T}$ - cell-mediated immunity. Out of primary variants, vitamin D2 and D3, the active form cholecaliferol (1, 25 dihydroxy vitamin D3), plays a noteworthy function in scientific medicine, thereby maintaining skeletal integrity. Due to its physiological functions, vitamin $\mathrm{D}$ is potent in preventing inflammatory bowel diseases, insulin-dependent diabetes, cardiovascular diseases, malignancies, and various autoimmune disorders and infectious diseases [1 - 4].

The ultraviolet rays from the sunlight are the primary source that endogenously synthesizes cholecalciferol on the skin surface. Animal sources, including dairy products or fatty fish and plant sources such as mushrooms, even 
contribute to vitamin D sources to a lower extent.

The reduced sunlight exposure attributed to melanin pigmentation, types of clothes worn by the individuals, and more importantly, solar zenith angle, latitude, seasonal variations, atmospheric pollution, insufficient intake of sources rich in vitamin $\mathrm{D}$ are the major etiologic factors associated with vitamin D deficiency states. From the clinically apparent manifestations ranging from rickets in children to periosteal reactions in adults; to the subclinical manifestations, vitamin D deficiency may lead to death in severely deficient individuals $[5,6]$.

The studies in literature reported $30-50 \%$ vitamin $\mathrm{D}$ deficient states in all age groups of countries like Europe, the United States, Canada, and New Zealand. In the past two decades, even in intense sunlight-prone countries like India, Iran, China, Saudi Arabia, etc., the studies have reported an estimated prevalence of vitamin $\mathrm{D}$ deficiency between 30 - 93\% of the population. Health care professionals play a major role in the evaluation of vitamin D deficient states in the different age groups of individuals and its systemic health implications [5, 7]. There are many studies on the Prevalence of vitamin D deficiency; however, very few published on its symptomatic correlation in the literature. Therefore, the present study was attributed to determine the Prevalence and symptomatic correlation of vitamin D deficiency in individuals visiting for an annual health checkup at a quaternary care hospital. Lucknow, Uttar Pradesh, India.

\section{Materials and Methods}

The present cross-sectional study was conducted in the department of Preventive health, at 330 bedded quaternary care Apollo Medics hospital in Lucknow, Uttar Pradesh. The approval from Medical Ethics Committee was taken before commencing the study. The written consent was obtained from all the participants. The study was conducted between February 2019 and April 2020 for the duration of 14 months.

\section{Study Population}

A total of 100 study participants reported for the annual health check-up to the department with prescribed serum vitamin D levels tests, and 91 met the inclusion criteria. Out of which, 56 were males, and 35 were females.

\section{Inclusion Criteria}

1. Patients between the age of $18-80$ years.

2. Both symptomatic and asymptomatic patients, prescribed with serum vitamin D level test.

3. Patients agreed to participate and sign for written consent.

\section{Exclusion Criteria}

1. Patients with a history of vitamin D supplements.

2. Patients with diagnosed hepatic or renal disorders.

3. Patients with diagnosed metabolic bone disorders.

4. Pregnant and lactating females.

5. Patients with diagnosed other systemic disorders like diabetes, hypercortisolism, etc.

\section{Data Collection and Questionnaire}

The patients visiting the department of Preventive health were enrolled after meeting the inclusion criteria. All the study participants were explained about the methodology, and consent were obtained. All questions were asked in the local language per the understanding of the study participants.

A structured questionnaire was used to record the demographic details and associated symptoms of the study participants. The demographic details include name, age, gender, weight, height, address, educational qualifications, socioeconomic status, etc. In the present study, symptoms were recorded as follows: 
1. Group 1 - Bony Symptoms: Joint pain, back pain associated with the spine, etc.

2. Group 2 - Muscular Symptoms: Myalgia, muscle weakness, muscle cramps, etc.

3. Group 3 - Generalized Symptoms: Weakness, lethargy, morning stiffness, etc.

4. Group 4 - Psychogenic symptoms: Depression, schizophrenia, mood swings, etc.

To maintain the uniformity, the symptoms were further graded according to severity (1 to 9 ), where the score 1-3 was categorized as mild, 4-6 as moderate, and 7-9 as severely symptomatic.

\section{Sample Collection and Processing}

The blood samples were collected from the study participants between 8:00 to 10:00 am for testing in NABH certified pathology laboratory at Apollo Medics hospital, Lucknow, Uttar Pradesh. The tests were performed using fully automatic machinery under the guidance of a senior pathologist. The deficient vitamin $\mathrm{D}$ levels were classified as -

1. Mild $=20-29 \mathrm{ng} / \mathrm{mL}$.

2. Moderate $=10-19 \mathrm{ng} / \mathrm{mL}$.

3. Severe $=<10 \mathrm{ng} / \mathrm{mL}$.
After obtaining demographic data, questionnaire records, and blood samples, the data was taken into a required format for further analysis. The descriptive data was mentioned in mean \pm standard deviation and percentages. To determine the significance of obtained data, the Chi-square test was used with a p-value less than 0.05 . The data recorded were tabulated and analyzed using software version 21 (SPSS Inc., Chicago, IL, USA).

\section{Results}

The Prevalence of study participants with vitamin D deficiency was $91 \%$ in the present study population. Out of 91 study participants enrolled in the study, $56(61.53 \%)$ were males, and $35(38.46 \%)$ were females. The majority of males and females had moderate levels of deficiency, followed by mild levels. The present study reveals no significant difference in both genders in terms of vitamin D deficient severity with a p-value of 0.707 . In the present study, the majority of study participants belong to the 5th decade of life, followed by the 4 th decade. The mean age of patients was $48.08 \pm$ 10.33 years in the present group of study participants (Table 1, Table 2, Figure 1).

Table 1. Age-wise Distribution of Study Participants according to the Severity of Vitamin D Deficient States

\begin{tabular}{|l|l|l|l|l|l|}
\hline Age Distribution & Mild & Moderate & Severe & Total & \multirow{2}{*}{ Percentage (\%) } \\
\hline $\mathbf{( y e a r s )}$ & $\mathbf{( 2 0} \mathbf{- 2 9} \mathbf{~ n g / m l )}$ & $\mathbf{( 1 0}-\mathbf{1 9} \mathbf{~ n g} / \mathbf{m l})$ & $\mathbf{( 1 - 9} \mathbf{~ n g} / \mathbf{m l})$ & $\mathbf{( n = 9 1 )}$ & \\
\hline $21-30$ & 2 & 2 & 1 & 5 & 5.49 \\
\hline $31-40$ & 3 & 7 & 5 & 15 & 16.48 \\
\hline $41-50$ & 13 & 14 & 4 & 31 & 34.06 \\
\hline $51-60$ & 14 & 17 & 1 & 32 & 35.16 \\
\hline $61-70$ & 3 & 4 & 0 & 7 & 7.69 \\
\hline $71-80$ & 0 & 0 & 1 & 1 & 1.09 \\
\hline Total & 35 & 44 & 12 & 91 & 100 \\
\hline
\end{tabular}

Table 2. Gender Wise Distribution of Study Participants

\begin{tabular}{|l|l|l|l|l|l|}
\hline \multirow{2}{*}{ Gender Distribution } & Mild & Moderate & Severe & Total & \multirow{2}{*}{ Percentage (\%) } \\
\cline { 2 - 6 } & $\mathbf{( 2 0} \mathbf{- 2 9} \mathbf{~ n g / m l )}$ & $\mathbf{( 1 0}-\mathbf{1 9} \mathbf{~ n g} / \mathbf{m l})$ & $\mathbf{( 1 ~ - 9 ~ \mathbf { ~ g } / \mathbf { m l } )}$ & $\mathbf{( n = 9 1 )}$ & \\
\hline Female & 15 & 15 & 5 & 35 & 38.46 \\
\hline Male & 20 & 29 & 7 & 56 & 61.53 \\
\hline
\end{tabular}




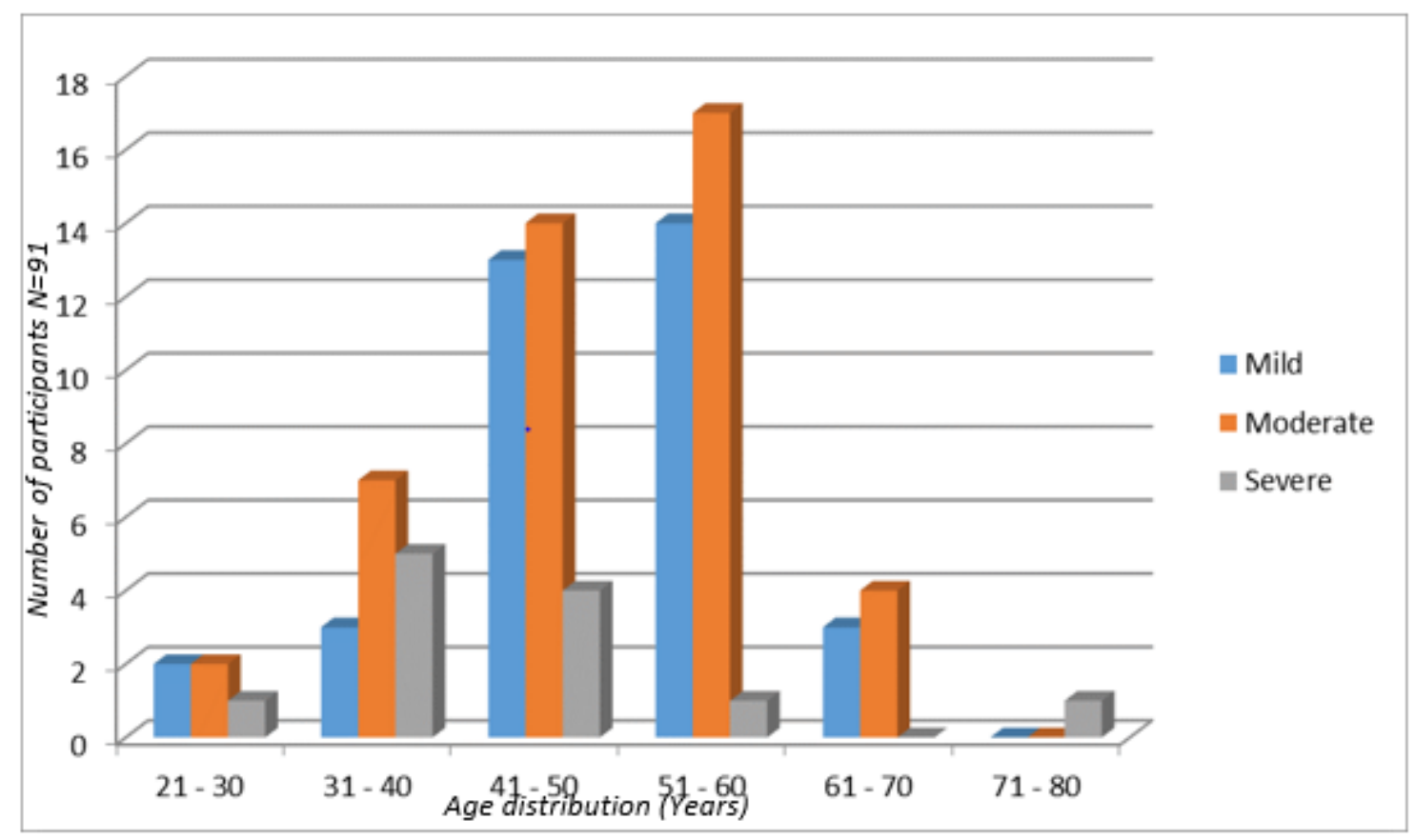

Figure 1. Age-wise Distribution of Symptomatic Study Participants

Out of 91 vitamin $\mathrm{D}$ deficient study participants, $83(91.2 \%)$ were symptomatic. Among $8(8.79 \%)$, asymptomatic patients only mild vitamin D deficiency was seen. On

applying chi-square test, the maximum numbers of individuals were in the moderate vitamin $\mathrm{D}$ deficiency category with p-value of 0.00 (Table 3).

Table 3. Vitamin D Deficiency - Severity Wise Distribution of Individuals

\begin{tabular}{|l|l|l|l|l|l|}
\hline \multirow{2}{*}{ Symptoms } & Mild & Moderate & Severe & Total & Percentage (\%) \\
\cline { 2 - 6 } & $\mathbf{( 2 0}-\mathbf{2 9} \mathbf{~ n g} / \mathbf{m l})$ & $\mathbf{( 1 0}-\mathbf{1 9} \mathbf{~ n g} / \mathbf{m l})$ & $\mathbf{( 1 - 9} \mathbf{~ g / m l )}$ & $\mathbf{( n = 9 1 )}$ & \\
\hline Present & 27 & 44 & 12 & 83 & 91.2 \\
\hline Absent & 8 & 0 & 0 & 8 & 8.79 \\
\hline
\end{tabular}

Bony symptoms such as joint pain or back pain were seen in the majority of study participants (75), followed by muscular (72) and generalized symptoms (71), while psychological symptoms were seen in 17 study participants only. On applying ANOVA test, there is a statistically significant difference between the occurrence of different symptoms while the psychological symptoms were found less compared to other symptoms (P-Value of < .00001) (Table 4).

Table 4. Symptoms Wise Distribution of Study Participants based on Severity

\begin{tabular}{|l|l|l|l|}
\hline \multirow{2}{*}{ Symptoms } & \multicolumn{3}{|l|}{ Study Participants (n) } \\
\cline { 2 - 4 } & $\begin{array}{l}\text { Mild Symptoms } \\
\text { (Score 1-3) }\end{array}$ & $\begin{array}{l}\text { Moderate Symptoms } \\
\text { (Score 4-6) }\end{array}$ & $\begin{array}{l}\text { Severe Symptoms } \\
\text { (Score 7-9) }\end{array}$ \\
\hline Bony Symptoms & 23 & 33 & 19 \\
\hline Muscular Symptoms & 20 & 30 & 22 \\
\hline General Symptoms & 15 & 29 & 27 \\
\hline Psychological Symptoms & 8 & 6 & 3 \\
\hline
\end{tabular}


The overall mean value of serum vitamin $\mathrm{D}$ is $17.54 \mathrm{ng} / \mathrm{mL}$, and the mean value of the total symptom score is 12.98 . The mild, moderate, and severe levels of serum vitamin D levels were seen in $32.53 \%, 53.01 \%$, and $14.45 \%$ of study participants, respectively. On applying the Pearson correlation coefficient formula, there is a strong negative correlation between the total symptom score and serum vitamin D levels with an R-value of -0.788 ( $\mathrm{p}$-value $=$ 1.84) (Figure 2).

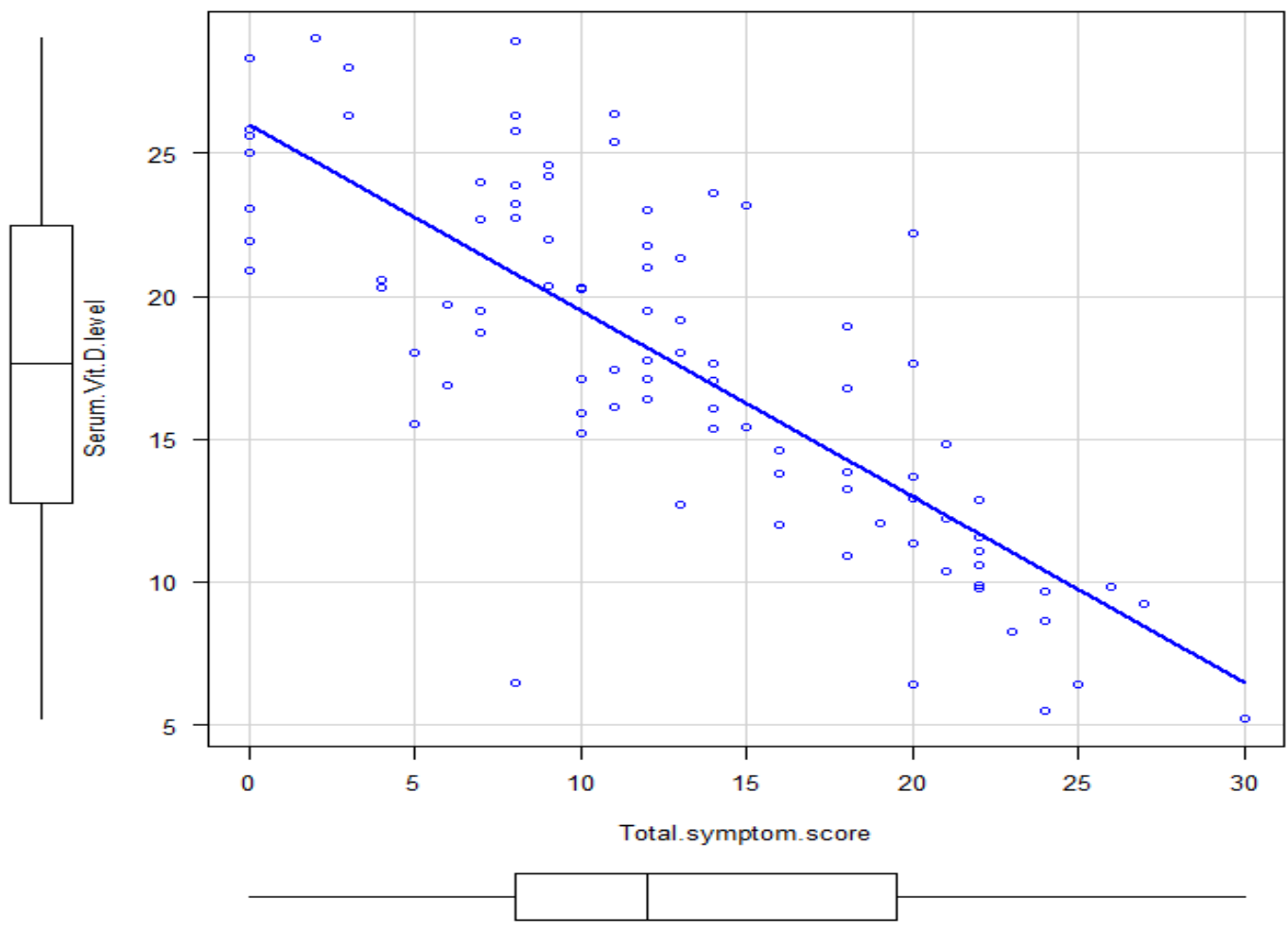

Figure 2. Correlation between Serum Vitamin D Levels and Total Symptom Score

All the symptoms have a moderate negative correlation with serum vitamin D levels except for psychological symptoms where there is a weak negative correlation. The mean serum values of vitamin $\mathrm{D}$ based on symptoms are listed in Table 5.

Table 5. The Mean Serum Vitamin D Levels based on Symptom Score

\begin{tabular}{|l|l|l|l|l|l|}
\hline \multirow{2}{*}{ Symptoms } & \multicolumn{2}{|l|}{ Mean Serum Vitamin D Levels (ng/ml) } \\
\cline { 2 - 7 } & $\begin{array}{l}\text { No } \\
\text { Symptoms } \\
\text { (Score 0) }\end{array}$ & $\begin{array}{l}\text { Mild } \\
\text { Symptoms } \\
\text { (Score 1-3) }\end{array}$ & $\begin{array}{l}\text { Moderate } \\
\text { Symptoms } \\
\text { (Score 4-6) }\end{array}$ & $\begin{array}{l}\text { Severe } \\
\text { Symptoms } \\
\text { (Score 7-9) }\end{array}$ & $\begin{array}{l}\text { Correlation of } \\
\text { Serum Vit D } \\
\text { with Symptom } \\
\text { Score (r value) }\end{array}$ \\
\hline Bony Symptoms & 20.72 & 22.52 & 15.94 & 11.61 & -0.6478 \\
\hline Muscular Symptoms & 20.15 & 15.95 & 16.42 & 18.28 & -0.5669 \\
\hline General Symptoms & 22.39 & 19.55 & 19.43 & 10.8 & -0.6933 \\
\hline $\begin{array}{l}\text { Psychological } \\
\text { Symptoms }\end{array}$ & 17.96 & 19 & 15.58 & 7.17 & -0.2775 \\
\hline
\end{tabular}




\section{Discussion}

In the current scenario, Vitamin D deficiency is considered one of the major medical conditions worldwide. The physiological variants of vitamin D2 and D3 play a significant role in clinical medicine ranging from maintaining skeletal and extra-skeletal integrity to its screenplay in supporting $\mathrm{T}$ cellmediated immunity. The noteworthy task of vitamin $\mathrm{D}$ in preventing various clinical and subclinical infections and disorders such as cardiovascular diseases, inflammatory bowel disease, malignancies, diabetes, metabolic disorders, etc., thereby proves its importance as a micronutrient. The major risk factors associated with vitamin D deficiency include inadequate sun exposure depending on geographical location, seasonal variations, atmospheric pollution, skin colour, types of clothing worn by population, and insufficient intake of vitamin D in terms of animal (fatty fish, fortified eggs) and plant (mushrooms) sources $[2,3,8,9]$.

With today's healthcare system and changing lifestyle in the 21st century, the deficient states of vitamin $D$ are seen in approximately 1 billion populations, according to global estimation. The Prevalence of vitamin $\mathrm{D}$ deficiency in the present study population is $91 \%$. A study has conducted by [10], in her review published in 2004, stated that the community and hospital-based studies in India have a prevalence rate of $50 \%$ to $94 \%$ and 37 $\%$ to $99 \%$, respectively, among healthy individuals. The authors further concluded that modernization, cultural practices, pollution, unplanned pregnancies, and increased use of sunscreen lotions are the major causes leading to vitamin $\mathrm{D}$ deficiency among all age groups. The above review is in accordance with the present study [10].

The reported Prevalence of vitamin D deficiency in healthy Indians is as high as $70 \%$ to $100 \%$ from all parts of the country, which is again in accordance with the present study. A similar study on vitamin D deficiency performed in the same city in 2004 on healthy individuals stated $78.3 \%$ deficient states. Another hospital-based cross-sectional study by [12] concluded a higher vitamin D deficiency prevalence rate among patients visiting the medical outpatient department in Mahad. The similarity between both the studies can be due to environmental factors, changing indoor lifestyles, insufficient nutritional intake, and lack of awareness among the population regarding the sources enriched in vitamin $D$ $[11,12]$.

In the present study, the mean age of study participants is $48.08 \pm 10.33$ years, with the majority of individuals belonging to the 4 th and 5 th decade of life. The study published by [13], stated that the potential risk of vitamin D deficiency is in direct association with increasing age. The reduced potential of vitamin $\mathrm{D}$ synthesis by the human body with age and lack of residential care leading to the insufficient synthesis of vitamin D in the body can be the associated factors suggesting a high prevalence rate of vitamin $\mathrm{D}$ deficiency in elderly individuals [13, 14]. The studies contradicting the present study was published by [15], stating the higher Prevalence of vitamin D deficiency in the adult population. The authors further supported the study by concluding that the increase in awareness regarding vitamin $\mathrm{D}$ supplements among the younger generation and changes in lifestyle such as clothing habits, reduced outdoor activities, etc [15]. Most of the studies published in the literature stated no significant variation in vitamin $\mathrm{D}$ deficiency among different age groups. This can be attributed to the similar age study participants included in the study thereby, further suggesting the need for comprehensive research on vitamin $\mathrm{D}$ deficiency among all age groups, ranging from children to the geriatric population [7].

The current study stated majority of males $(61.53 \%)$ are in the present deficient population. [13] included only males in the 
study determining the Prevalence of osteoporosis in the aged 50 years and above population. He concluded the severity of vitamin $\mathrm{D}$ deficiency and osteoporosis is more relevant in females as per literature, but the present study stated that the males are even equally deficient in vitamin D [13]. Another case-control study conducted on 120 males suffering from acute myocardial infarction seeking treatment in AIIMS, Delhi, concluded the deficiency of vitamin D is strongly associated with cardiovascular diseases in males [16]. The above-stated studies showed higher Prevalence in males as per the present study, while various studies in literature also showed the greater Prevalence of vitamin D deficiency among pregnant and postmenopausal women [10].

The mean serum levels of vitamin $\mathrm{D}$ in the present study participants are $17.54 \mathrm{ng} / \mathrm{mL}$. In the present study, the mild $(20-29 \mathrm{ng} / \mathrm{mL})$, moderate (10-19 $\mathrm{ng} / \mathrm{mL})$ and severe $(<10 \mathrm{ng} / \mathrm{mL})$ levels of serum vitamin D levels were seen in $32.53 \%, 53.01 \%$ and $14.45 \%$ of study participants respectively. The serum levels $<20 \mathrm{ng} / \mathrm{mL}$ were seen in $67.46 \%$ of study participants, which is in accordance with the below studies published in the literature. A review published by Aparna $\mathrm{P}$ involving the community and hospital-based vitamin D deficiency prevalence studies considers the serum $25(\mathrm{OH}) \mathrm{D}$ levels less than $20 \mathrm{ng} / \mathrm{mL}$ as deficient [10]. Other hospital-based studies published by $[12,17]$ among the Indian population stated that similar levels of serum vitamin D levels $(<20 \mathrm{ng} / \mathrm{mL})$ were seen in $65.4 \%$ and $70 \%$ of study participants, respectively. A similar study conducted by Roy A et al. among AIIMS, Delhi study participants were stating $<10 \mathrm{ng} / \mathrm{mL}$ and $10-30 \mathrm{ng} / \mathrm{mL}$ serum vitamin D levels among 79.2 \%and 19.2 $\%$ cases, respectively, enrolled in the study [16]. Another Delhi based hospital study in consonance with the present study, published by [18] among healthy individuals (above 50 years) stated that the severe vitamin $\mathrm{D}$ deficient levels of $<20 \mathrm{ng} / \mathrm{mL}$ were seen among $91.2 \%$ study participants and serum levels between $20-30 \mathrm{ng} / \mathrm{mL}$ were seen in $6.8 \%$ participants only [18].

The present study revealed that general (78.02\%), bony $(82.41 \%)$, and muscular (79.12\%) symptoms were seen in the majority of the study participants, which is in consonance with the study published in literature [19, 20]. [19] conducted a study on 276 individuals with musculoskeletal pain and further concluded that $63.4 \%$ of participants were diagnosed with vitamin D deficiency. [20] conducted a study on 150 participants diagnosed with musculoskeletal pain, in which $93 \%$ of patients had vitamin D deficiency. Another published research by Stewart and Hirani concluded that depressive symptoms are strongly associated with clinical vitamin D deficiency, which is not in accordance with the present study stating that only $18.68 \%$ of study participants complain of psychological symptoms [21]. A strong association between vitamin D deficiency and musculoskeletal symptoms was seen in present study participants, while psychological symptoms showed a weak correlation. Though the majority $(91 \%)$ seems to be symptomatic, these people didn't present to the hospital for these symptoms. The present study is single centered along with the limited number of study participants enrolled. In the future, we recommend the multi-centred study in the same geological location, along with a large study population. The study also reveals the need for a validated questionnaire and clinical scoring system to evaluate the signs and symptoms associated with vitamin D deficiency.

\section{Conclusion}

The present study concluded that vitamin D deficiency is heading towards the silent epidemic. This stresses on increase in awareness regarding the advantages of routine sunlight exposure and intake of fortified rich sources. Previously, the increased burden on 
health care professionals was more associated with compromised individuals, but in the present 21 st century, the alarming prevalence rate of $91 \%$ among healthy individuals has raised a question on the modernization, insufficient dietary intake, and lastly, on the country's health care system. With the known consequences of its deficiency, the present condition needs due attention through comprehensive public health programs.

\section{Acknowledgement}

I acknowledge the generous support from the Apollo medics hospital administration for providing all support in planning and conducting the study in the Department of Preventive Health.

\section{References}

[1] Nair R, Maseeh A. 2012, Vitamin D: "The Sunshine" Vitamin. J Pharmacol Pharmacother; 3:118-26.

[2] Holick MF, Chen TC. 2008, Vitamin D deficiency: a worldwide problem with health consequences. Am J Clin Nutr.;87:1080S-6S.

[3] Heaney RP, Dowell MS, Hale CA, Bendich A. 2003, Calcium absorption varies within the reference range for serum 25-hydroxyvitamin D. $J$ Am Coll Nutr.;22:142-6.

[4] Holick MF. 2004, Sunlight and vitamin D for bone health and prevention of autoimmune diseases, cancers, and cardiovascular disease. J Am Clin Nutr. 1; 80:1678S-88S.

[5] Tabrizi R, Moosazadeh M, Akbari M, Dabbaghmanesh MH, Mohamadkhani M, Asemi Z, Heydari ST, Akbari M, Lankarani KB. 2018, High Prevalence of Vitamin D deficiency among Iranian population: a systematic review and meta-analysis. Iran J Med Sci.; 43(2):125-39.

[6] Holick MF. 2006, High Prevalence of vitamin D inadequacy and implications for health. Mayo Clin Proc.:81; 353-73.

[7] Hovsepian S, Amini M, Aminorroaya A, Amini P, Iraj B. 2011, Prevalence of vitamin D deficiency
I am indebted to my head of department, Prof. Dr. RC Ahuja, for his continued guidance. His honest approach to medical research is a source of inspiration.

Thank you to my colleague Dr. Rakhi for her support in data analytics and write-ups. I am thankful to the Department of Pathology \& Biochemistry for giving quality lab reports timely. And last but not least, I am grateful to all preventive health staff especially, Rahul, Kamal, Kalpana, Sunayna \& Mamta, for their support in facilitating sample collection and report collection and compilation.

\section{Conflict of Interest}

We have no conflict of interest.

among adult population of Isfahan City, Iran. J Health Popul Nutr.; 29:149-55.

[8] Hickey L, Gordon CM. 2004, Vitamin D deficiency: new perspectives on an old disease. Curr Opin Endocrinol Diabetes.; 11:18-25.

[9] Lips P. 2007, Vitamin D status and nutrition in Europe and Asia. J Steroid Biochem Mol Biol.; 103:620-5.

[10] Aparna P, Muthathal S, Nongkynrih B, Gupta SK. 2018, Vitamin D deficiency in India. J Family Med Prim Care.; 7(2):324-30.

[11]Lips P. 2001, Vitamin D deficiency and secondary hyperparathyroidism in the elderly: consequences for bone loss and fractures and therapeutic implications. Endocrine reviews.; 22:477-501.

[12] Bawaskar PH, Bawaskar HS, Bawaskar PH, Pakhare AP. 2017, Profile of Vitamin D in patients attending at general hospital Mahad India. Indian $J$ Endocrinol Metab; 21(1):125-30.

[13] Agrawal NK, Sharma B. 2013, Prevalence of osteoporosis in otherwise healthy Indian males aged 50 years and above. Arch Osteoporos.; 8:116.

[14] Parva NR, Tadepalli S, Singh P, Qian A, Joshi R, Kandala H, Nookala VK, Cheriyath P. 2018, Prevalence of vitamin D deficiency and associated 
risk factors in the US population (2011-2012). Cureus.;10(6):2741.

[15]Rattan R, Sahoo D, Mahapatra S. 2016, Prevalence of Vitamin D deficiency in adults in the coastal regions of Odisha, India. IOSR J Pharm Biol Sci.; 11:49-52.

[16] Roy A, Lakshmy R, Tarik M, Tandon N, Reddy KS, Prabhakaran D, et al. 2015, Independent Association of severe Vitamin D deficiency as a risk of acute myocardial infarction in Indians. Indian Heart J.; 67:27-32.

[17] Goswami R., Kochupillai N., Gupta N. 2008, Presence of $25(\mathrm{OH})$ D deficiencies in a rural North Indian village despite abundant sunshine. $J$ Assoc Physicians India; 56:755-7.
[18] Marwaha R.K., Tandon N., Garg M.K. 2011, Vitamin D status in healthy Indians aged 50 years and above. J Assoc Physicians India.; 59:706-9.

[19] Heidari B, Shirvani J. S, Firouzjahi A, Heidari P, Hajian-Tilaki K. O. 2010, association between nonspecific skeletal pain and vitamin D deficiency. Int J Rheum Dis.;13(4):340-6.

[20] Plotnikoff GA, Quigley JM. 2003, Prevalence of severe hypovitaminosis $\mathrm{D}$ in patients with persistent, nonspecific musculoskeletal pain. Mayo Clin Proc; 78(12):1463-70.

[21] Stewart R, Hirani V. 2010, Relationship between vitamin $\mathrm{D}$ levels and depressive symptoms in older residents from a national survey population. Psychosom Med.; 72(7):608-12. 